\title{
Anti-Tim4 Grafting Strongly Hydrophilic Metal-Organic Frameworks Immunoaffinity Flake for High-Efficiency Capture and Separation of \\ Exosomes
}

Lei Zhang ${ }^{\mathrm{a}, \mathrm{b}, \mathrm{c}}$, Haiyan Wang ${ }^{\mathrm{a}, \mathrm{d}}$, Guofa Zhao ${ }^{\mathrm{b}, \mathrm{c}}, \mathrm{Nan} \mathrm{Li}^{\mathrm{b}, \mathrm{c}}$, Xiaofang Wang ${ }^{\mathrm{b}, \mathrm{c}}$, Yumiao $\mathrm{Li}^{\mathrm{b}, \mathrm{c}}$, Youchao Jia ${ }^{\mathrm{b}, \mathrm{c}_{*}}$, Xiaoqiang Qiao ${ }^{\mathrm{a}, \mathrm{d}_{*}}$

${ }^{a}$ College of Pharmaceutical Sciences, Key Laboratory of Public Health Safety of Hebei Province, Hebei University, Baoding 071002, China

${ }^{\mathrm{b}}$ Department of Medical Oncology, Hebei Key Laboratory of Cancer Radiotherapy and Chemotherapy, Affiliated Hospital of Hebei University, Baoding 071000, China

${ }^{\mathrm{c}}$ College of Clinical Medicine, Hebei University, Baoding 071002, China

${ }^{\mathrm{d}}$ Key Laboratory of Medicinal Chemistry and Molecular Diagnosis, Ministry of Education, Institute of Life Science and Green Development, Hebei University, Baoding 071002, China

\section{Corresponding author:}

*Professor Dr. Youchao Jia, Department of Medical Oncology, Hebei Key Laboratory of Cancer Radiotherapy and Chemotherapy, Affiliated Hospital of Hebei University, Baoding, 071000, China

Tel.: +86-15933760921

E-mail: youchaojia@163.com

*Professor Dr. Xiaoqiang Qiao, College of Pharmaceutical Sciences, Key Laboratory of Public Health Safety of Hebei Province, Hebei University, Baoding, 071002, China Tel.: +86-312-5073196

E-mail: hbuqiao@163.com; xiaoqiao@hbu.edu.cn 
Table of contents

\section{Supplementary Experimental Section}

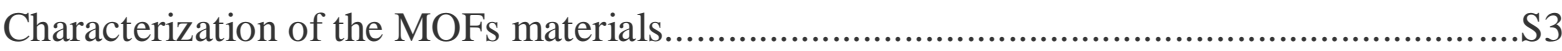

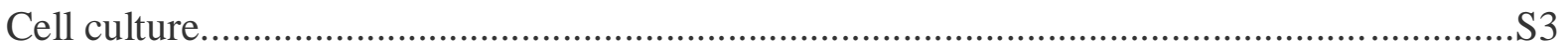

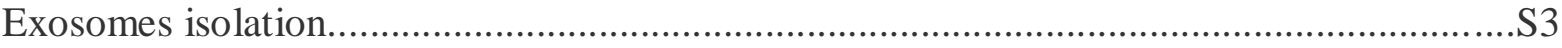

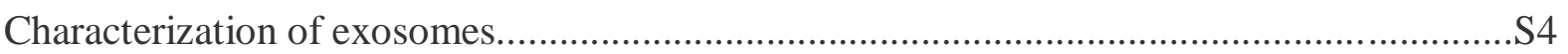

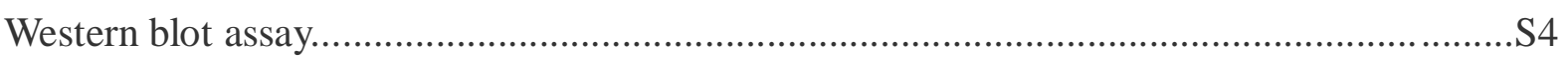

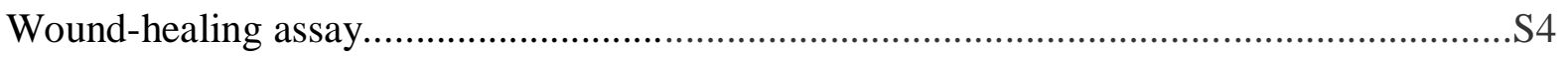

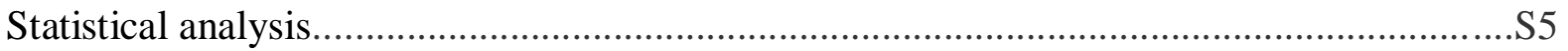

\section{Supplementary Figure}

Figure S1: Bright and fluorescent images of antibody functionalization.............................S6

Figure S2: Non-specific adsorption experiment of the ILI-01 MOFs material......................S6

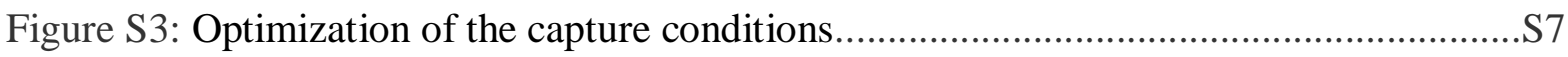

Figure S4: Fluorescent images of exosomes capture and release process.............................S7

Figure S5: Capture efficiencies of exosomes for different cells.........................................S8

Figure S6: Comparison of exosomes isolated via the developed Tim4@ILI-01

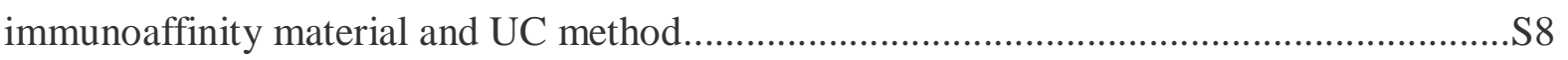

Figure S7: Comparison of the Tim4@ILI-01, CD63@ILI-01, and CD81@ILI-01 MOFs

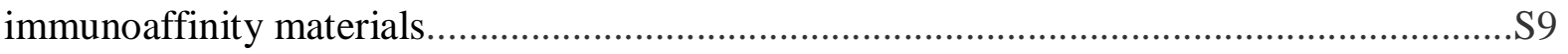

Figure S8: Western blot results of the expressed EMT-related proteins of H1299 cells

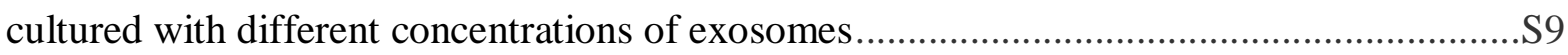

Figure S9: Effect of incubation time on the expression of EMT-related proteins of H1299

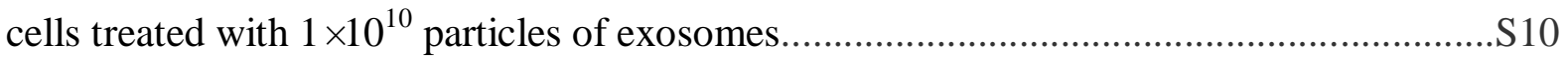




\section{Experimental Section}

\section{Characterization of the MOFs materials}

FT-IR spectra were obtained from Bruker Vertex 70 spectrometer (Ettlingen, Germany). XRD signals were determined via Ni-filtered $\mathrm{Cu} \mathrm{K} \alpha$ irradiation $(\lambda=1.5406 \AA$ ) with Bruker D8 X-ray diffractometer (Karlsruhe, Germany). SEM pictures were obtained via Gemini SEM 300 system (Carl Zeiss, Jena, Germany). Fluorescent images were obtained using a Leica inverted fluorescence microscope (Wetzlar, Germany). Water contact angle was measured via Dataphysics OCA 15EC system (Stuttgart, Germany).

\section{Cell culture}

H1299 (human lung adenocarcinoma cell) was grown in RPMI-1640 medium with 10\% (v/v) FBS, using a humidified incubator at $37{ }^{\circ} \mathrm{C}$ with $5 \% \mathrm{CO}_{2}$. When they reached $90 \%$ confluency, the cells were digested with trypsin-EDTA solution, followed by centrifugation at $1400 \mathrm{rpm}$ for $4 \mathrm{~min}$. The collected cells were split for further culturing. MGC-803 (human gastric cancer cell) and MDA-MB-231 (human breast cancer cell) were grown in DMEM medium with $10 \%$ (v/v) FBS. The other operations were the same as that for the H1299 cell.

Before exosomes extraction, the cell culture supernatant was sequentially centrifuged at $500 \times \mathrm{g}$ for $5 \mathrm{~min}, 2000 \times \mathrm{g}$ for $20 \mathrm{~min}$, and further filtered through $0.22 \mu \mathrm{m}$ filter, ready for use.

\section{Exosomes isolation}

For exosomes isolation via CD63@ILI-01 or CD81@ILI-01 immunoaffinity materials, 8 mg of immunoaffinity material was added into $4 \mathrm{~mL}$ cell culture supernatant and the mixture was stirred gently for $2 \mathrm{~h}$ at $4{ }^{\circ} \mathrm{C}$. The immunoaffinity material was centrifuged at $5500 \mathrm{rpm}$ for $5 \mathrm{~min}$ and continuously washed three times with $1 \mathrm{~mL}$ of PBS buffer. The immunoaffinity material was redissolved in $100 \mu \mathrm{L}$ PBS buffer and the bound exosomes were lysed by 100 $\mu \mathrm{L}$ RIPA buffer for downstream analysis. 
For exosomes isolation via the UC method, the cell culture supernatant was precipitated via centrifugation at $100,000 \mathrm{~g}$ for $70 \mathrm{~min}$. The obtained exosomes were further resuspended in $100 \mu \mathrm{L}$ PBS buffer and stored at $-80{ }^{\circ} \mathrm{C}$ for further analysis.

\section{Characterization of exosomes}

The TEM pictures of exosomes were obtained using a JEOL JEM-100SX instrument (Tokyo, Japan). Exosomes were firstly fixed and deposited on copper grids with $4 \%$ paraformaldehyde. Then, the samples were negatively stained with $2 \%$ phosphotungstic acid solution at $100 \mathrm{kV}$ for 3-5 min.

The concentration and size ranges of exosomes were measured using NanoSight NS500 (Malvern, UK). Exosomes were diluted 500-fold with PBS buffer and then loaded into NanoSight NS500. The scatter lights were collected by a high-sensitivity CMOS camera.

\section{Western blot assay}

The proteins were firstly extracted with RIPA buffer containing protease-inhibitor. The protein concentration was determined by using BCA Protein Assay Kit. The proteins were loaded and further separated with 10-12\% gradient SDS-PAGE, followed by transferring onto PVDF membranes. The PVDF membranes were further incubated with $4 \%$ BSA in TBST buffer at $37{ }^{\circ} \mathrm{C}$ for $2 \mathrm{~h}$ and then washed with TBST buffer three times for $10 \mathrm{~min}$. The primary antibodies were diluted (1:500-1:1000) in TBST buffer and then applied to the PVDF membranes at $4{ }^{\circ} \mathrm{C}$ overnight. After further washing with TBST buffer for three times, the PVDF membranes were incubated at the HRP-conjugated anti-mouse IgG antibody or HRP-conjugated anti-rabbit IgG antibody (1:5000) for $1 \mathrm{~h}$ at room temperature. After further washing with TBST buffer for three times, the signals were detected using ECL and a ChemiDoc system.

\section{Wound-healing assay}


For wound-healing assay, H1299 cells were cultured in the six-well plates overnight. When the confluency reached $100 \%$, the cell monolayer was scratched with a pipet tip, followed by washing twice with PBS buffer so as to remove the detached cells. The scratched cells were further treated with $2 \mathrm{~mL}$ of FBS-free cell culture medium containing $1 \times 10^{2}, 1 \times 10^{4}, 1 \times 10^{6}$, $1 \times 10^{8}$, or $1 \times 10^{10}$ particles of exosomes, respectively. The control experiment was also carried out without adding exosomes under the same conditions. The wound widths were measured using a microscope under culturing at 0,24 and $36 \mathrm{~h}$. The wound area was calculated via the software of Image J.

\section{Statistical analysis}

All data were presented by mean \pm standard deviation $(\mathrm{SD})$ of independent experiments. Statistical analysis was assessed by one-way ANOVA. The comparisons of the experimental setting between two groups were performed using the unpaired t-test. P-values $<0.05$ was considered to be statistically significant. Data analysis was performed by GraphPad Prism 6.0 statistical program. 


\section{Supplementary Figure}
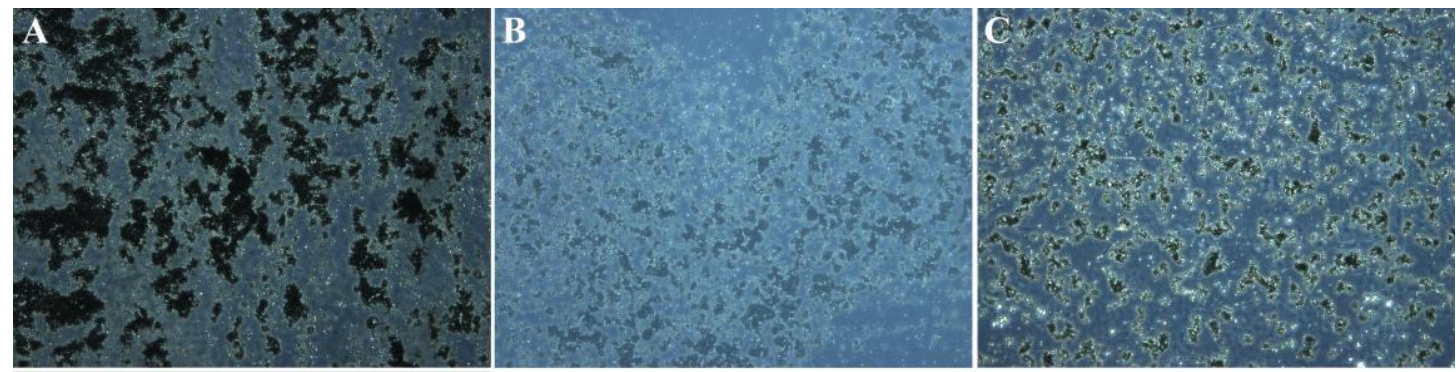

D

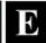

F

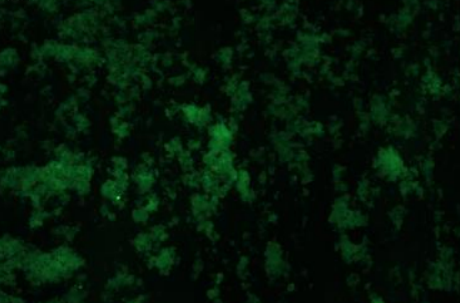

E

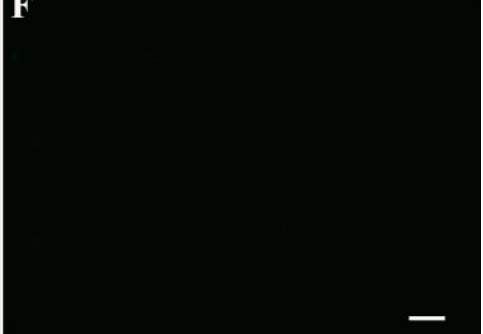

Figure S1 Bright and fluorescent images of Tim4@ILI-01 immunoaffinity material (A, D) and ILI-01 MOFs material (B, E) incubated with FITC-labeled goat anti-mouse IgG as well as the control Tim4@ILI-01 immunoaffinity material (C, F) (excitation 362 nm, emission 488 nm). Scale bar: $1 \mu \mathrm{m}$.

A

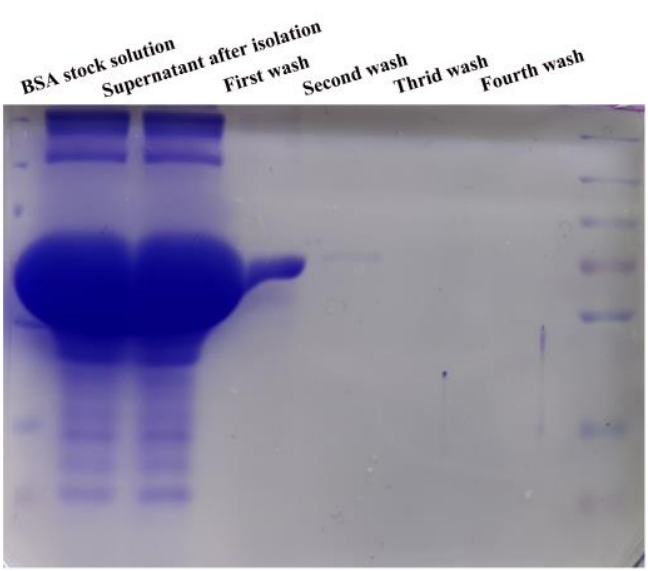

B

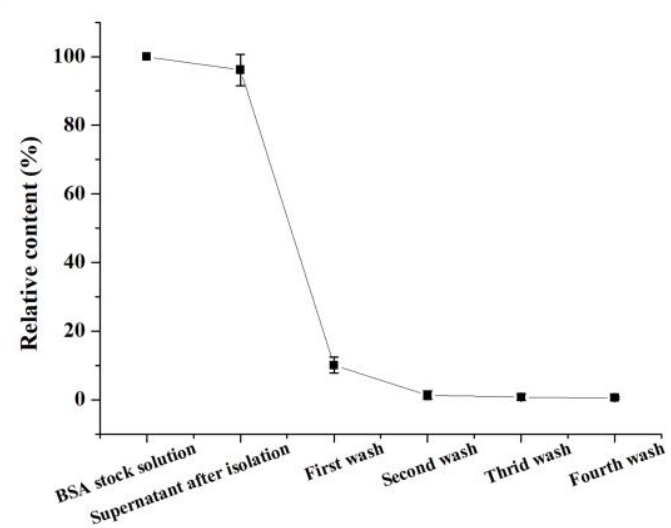

Figure S2 Non-specific adsorption experiment of the ILI-01 MOFs material: (A) SDS-PAGE characterization (BSA as the standard); (B) BCA assay of non-specific adsorption. 
A

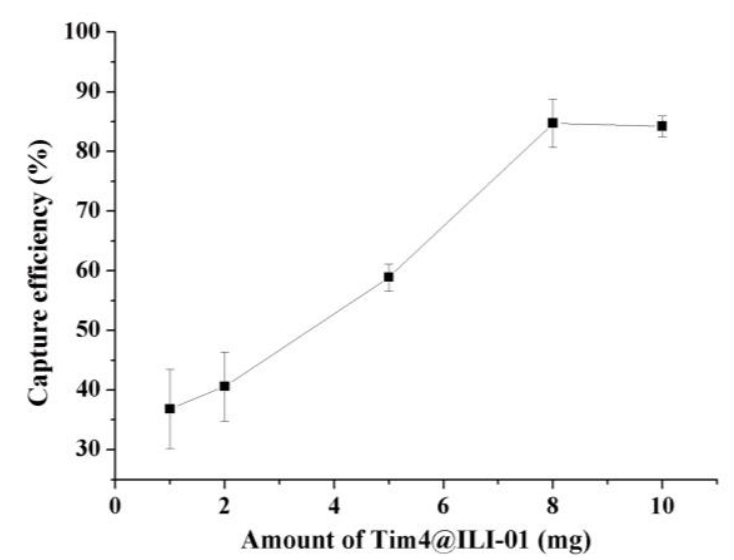

B

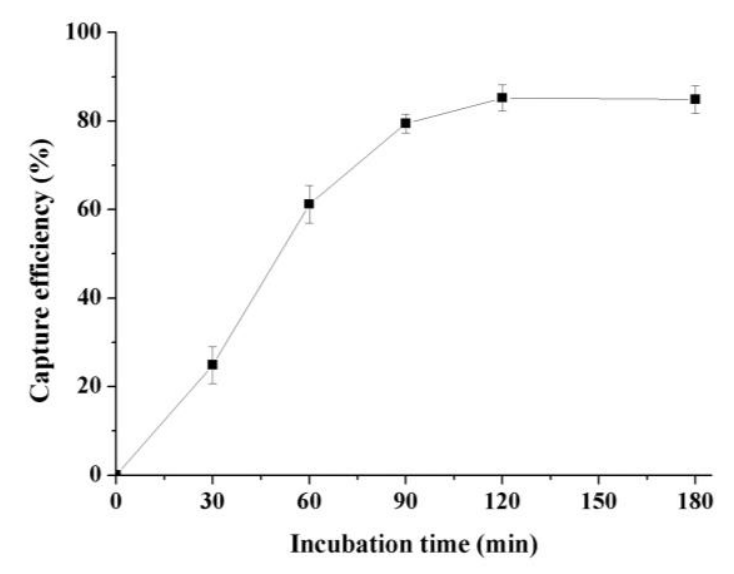

Figure S3 Optimization of the capture conditions: the effects of the amount of the Tim4@ILI-01 immunoaffinity material (A) and incubation time (B).

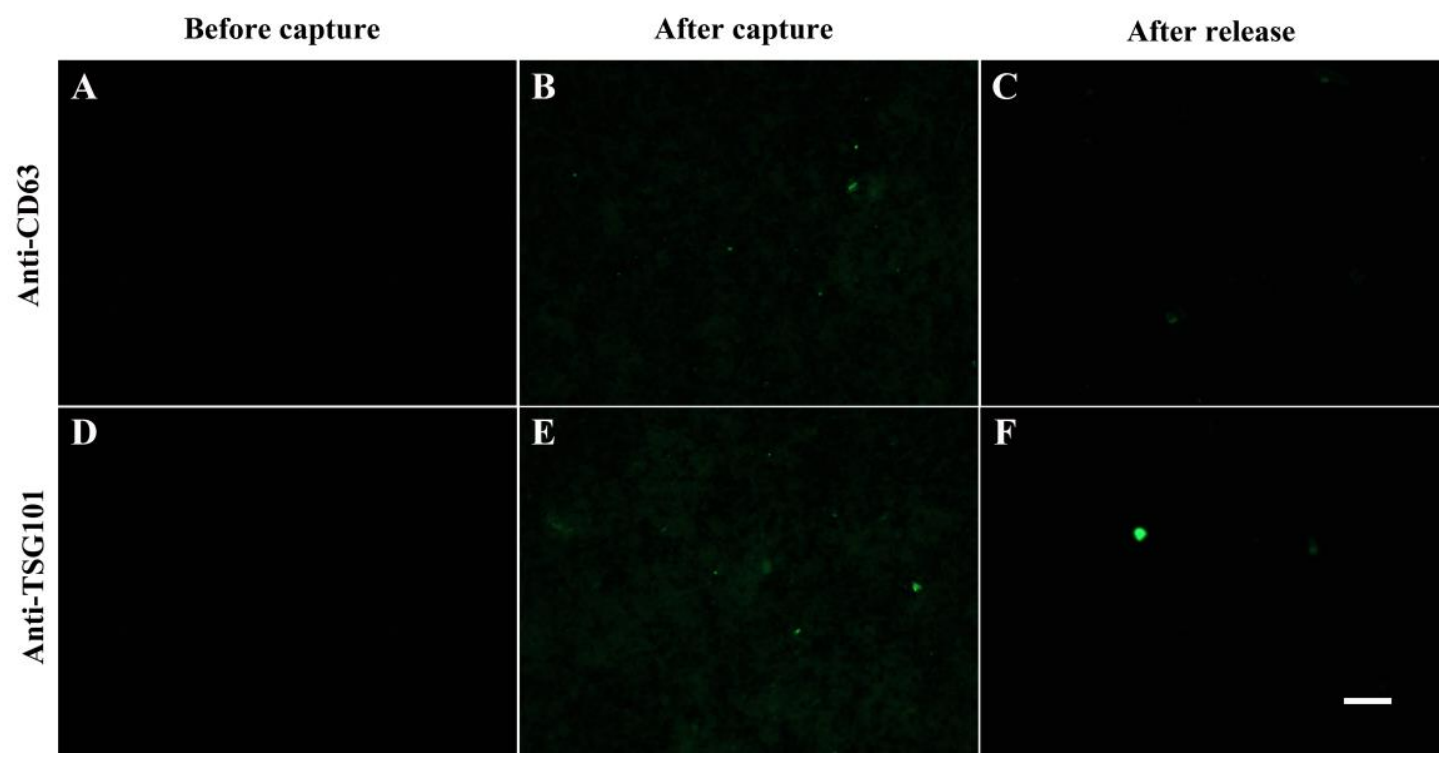

Figure S4 Fluorescent images of exosomes capture and release process: (A, D) Tim4@ ILI-01 immunoaffinity material; (B, E) Tim4@ILI-01 immunoaffinity material capturing with exosomes; (C, F) The released exosomes in the eluent. All the samples were prestained with anti-CD63 or anti-TSG101 antibodies and further incubated with green FITC-labeled goat anti-rabbit IgG. Scale bar: $1 \mu \mathrm{m}$. 


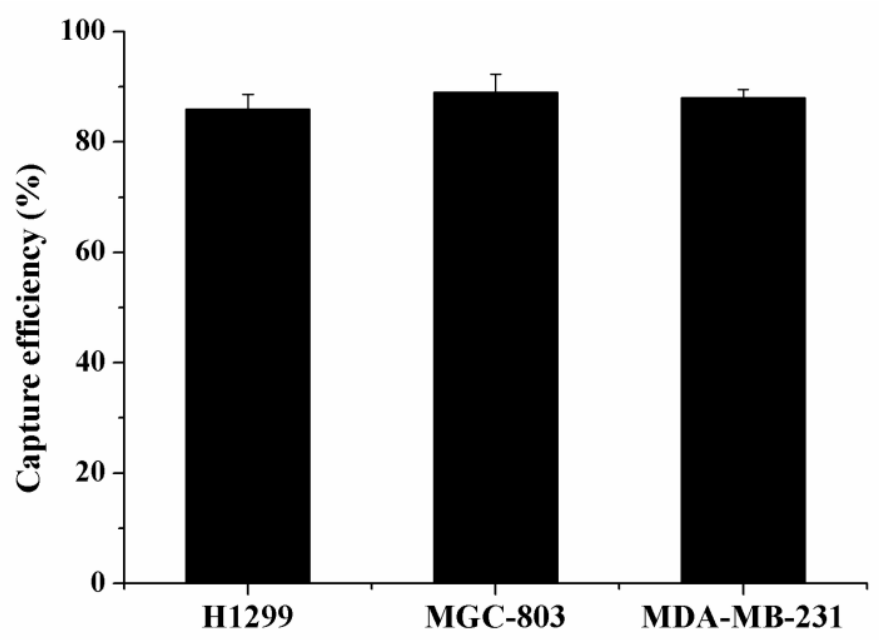

Figure S5 Capture efficiencies of exosomes for H1299, MGC-803, and MDA-MB-231 cells.

A

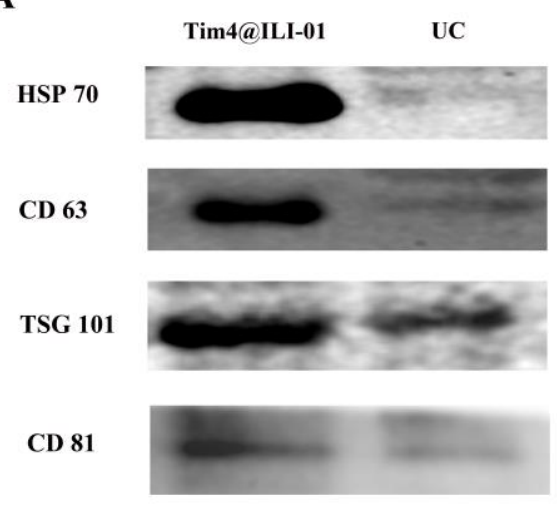

B

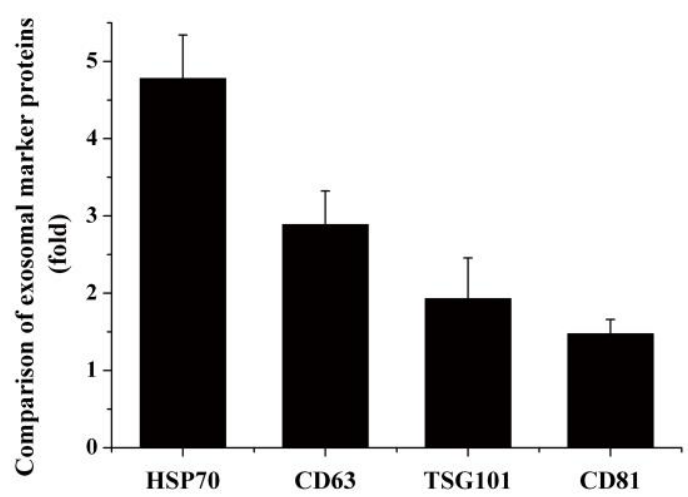

Figure S6 Comparison of exosomes isolated via the developed Tim4@ILI-01 immunoaffinity material and UC method: (A) Western blot results of exosomes marker proteins; (B) Comparison of the protein amounts. 
A

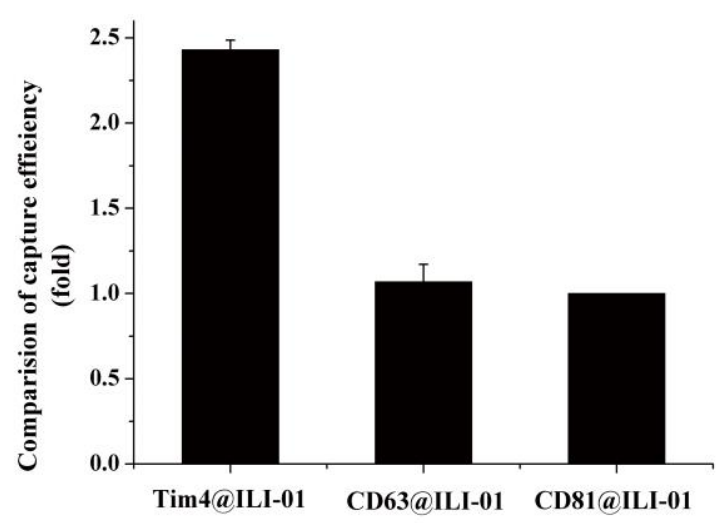

B

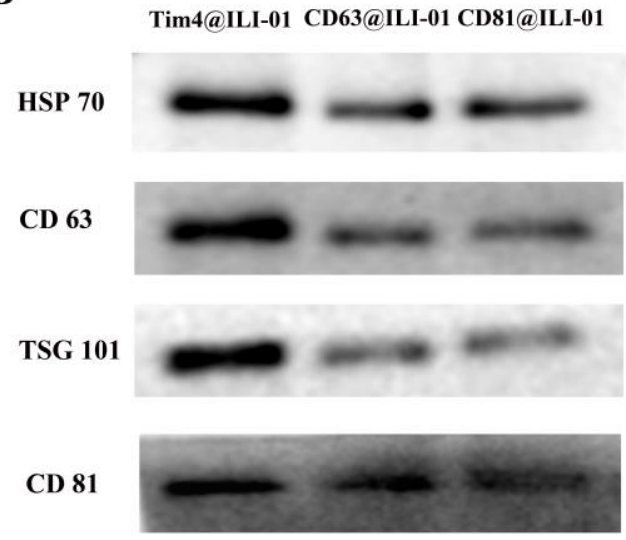

Figure S7 Comparison of the Tim4@ILI-01, CD63@ILI-01, and CD81@ILI-01 MOFs immunoaffinity materials: (A) Capture efficiency; (B) Western blot analysis of exosomes marker proteins.

A

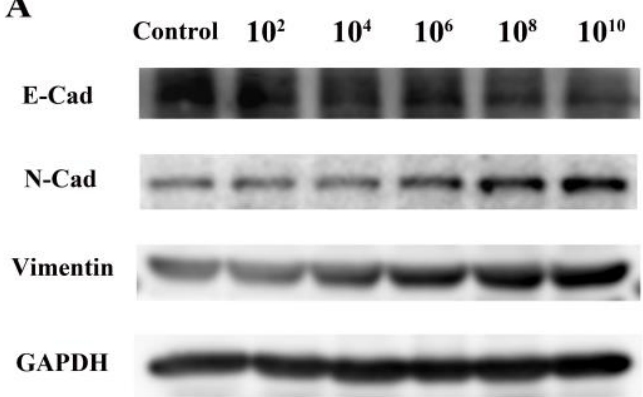

C

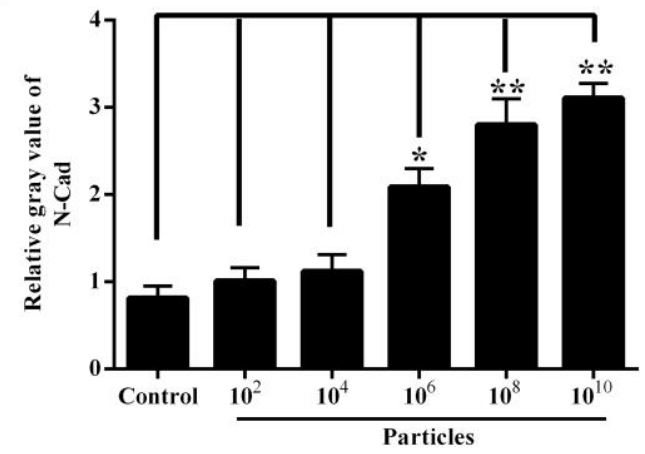

$\mathbf{B}$

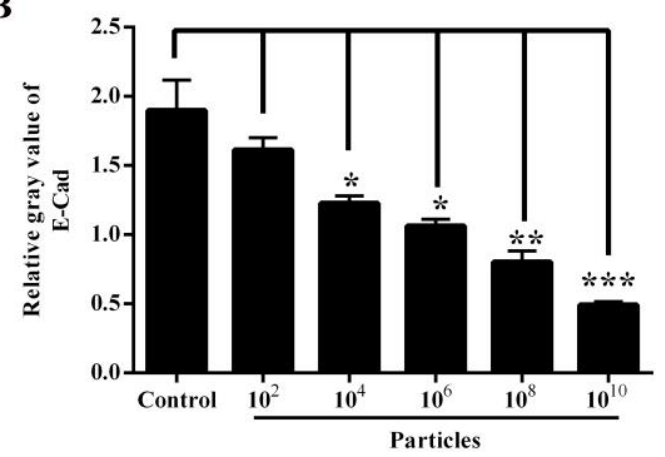

D

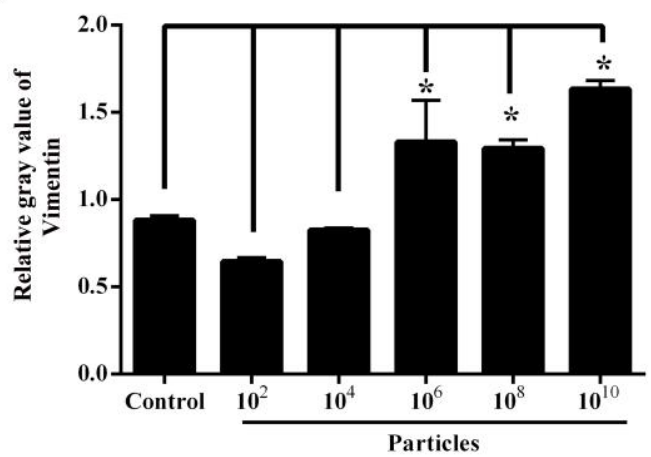

Figure S8 Western blot results of the expressed EMT-related proteins of H1299 cells cultured with different concentrations of exosomes. Data are reported as mean $\pm \mathrm{SD}, \mathrm{n}=3$ in each group. ${ }^{*} p<0.05$ versus Control; $* * p<0.01$ versus Control; $* * * p<0.001$ versus Control. 


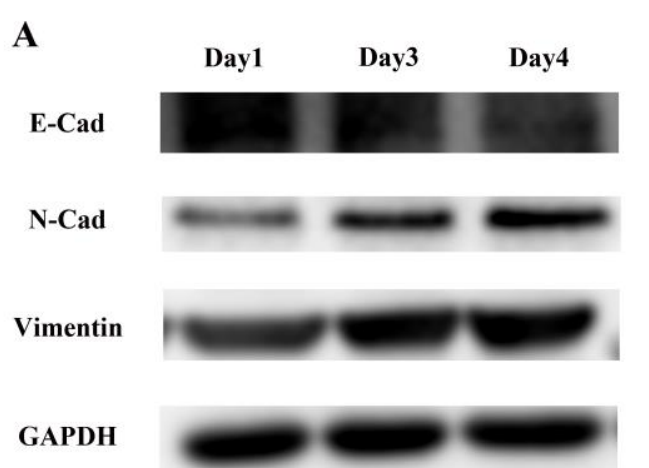

C

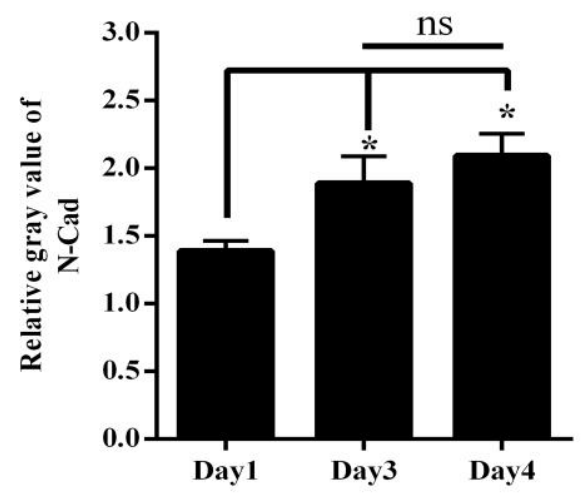

B

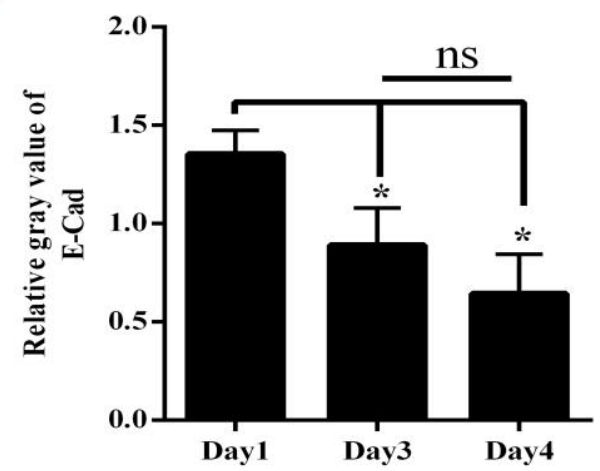

D

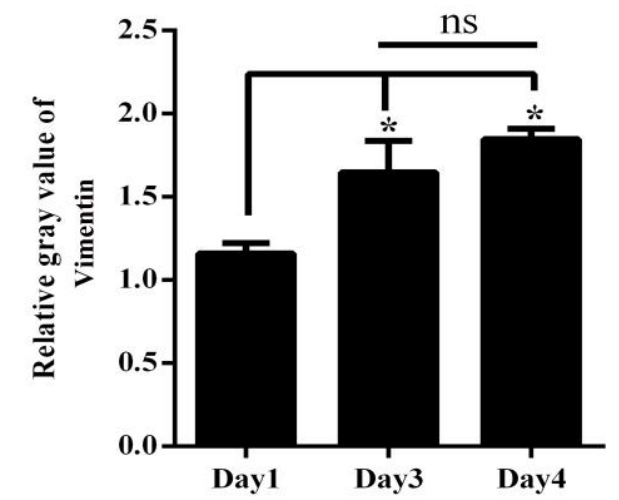

Figure S9 Effect of incubation time on the expression of EMT-related proteins of H1299 cells treated with $1 \times 10^{10}$ particles of exosomes. Data are reported as mean $\pm \mathrm{SD}, \mathrm{n}=3$ in each group. $* p<0.05$ versus Day 1. 